\title{
Article
}

\section{Category Algebras and States on Categories}

\author{
Hayato Saigo
}

check for

updates

Citation: Saigo, H. Category Algebras and States on Categories. Symmetry 2021, 13, 1172. https:// doi.org/10.3390/sym13071172

Academic Editors: Motoichi Ohtsu and Alexey Kanel-Belov

Received: 28 May 2021

Accepted: 26 June 2021

Published: 29 June 2021

Publisher's Note: MDPI stays neutral with regard to jurisdictional claims in published maps and institutional affiliations.

Copyright: (C) 2021 by the authors. Licensee MDPI, Basel, Switzerland. This article is an open access article distributed under the terms and conditions of the Creative Commons Attribution (CC BY) license (https:// creativecommons.org/licenses/by/ $4.0 /)$.
Nagahama Institute of Bio-Science and Technology, 1266 Tamura, Nagahama, Shiga 526-0829, Japan; h_saigoh@nagahama-i-bio.ac.jp

Abstract: The purpose of this paper is to build a new bridge between category theory and a generalized probability theory known as noncommutative probability or quantum probability, which was originated as a mathematical framework for quantum theory, in terms of states as linear functional defined on category algebras. We clarify that category algebras can be considered to be generalized matrix algebras and that the notions of state on category as linear functional defined on category algebra turns out to be a conceptual generalization of probability measures on sets as discrete categories. Moreover, by establishing a generalization of famous GNS (Gelfand-Naimark-Segal) construction, we obtain a representation of category algebras of ${ }^{\dagger}$-categories on certain generalized Hilbert spaces which we call semi-Hilbert modules over rigs. The concepts and results in the present paper will be useful for the studies of symmetry/asymmetry since categories are generalized groupoids, which themselves are generalized groups.

Keywords: category; algebra; state; category algebra; state on category; noncommutative probability; quantum probability; GNS representation

\section{Introduction}

In the present paper, we study category algebras and states defined on arbitrary small categories to build a new bridge between category theory (see [1-4] and references therein, for example) and noncommutative probability or quantum probability (see [5-7] and references therein, for example), a generalized probability theory which was originated as a mathematical framework for quantum theory.

A category algebra is, in short, a convolution algebra of functions on a category. For example, on certain categories called finely finite category [8], which is a categorical generalization of locally finite poset, the convolution operation can be defined on the set of arbitrary functions and it becomes a unital algebra called incidence algebra. Many authors have studied the notions of Möbius inversion, which has been one of fundamental part of combinatorics since the pioneering work by Rota [9] on posets, in the context of incidence algebras on categories ([8,10-14], for example).

There is another approach to obtain the notion of category algebra. As is well known, a group algebra is defined as a convolution algebra consisting of finite linear combinations of elements. By generalization with replacing "elements" by "arrows", one can obtain another notion of category algebra (see [13], for example), which also includes monoid algebra (in particular polynomial algebras) and groupoid algebras as examples. Please note that for a category with infinite number of objects, the algebra is not unital.

The category algebras we focus on in the present paper are unital algebras defined on arbitrary small categories, which are slightly generalized versions of algebras studied under the name of the ring of an additive category [15]. These category algebras include the ones studied in [13] as subalgebras in general, and they coincide for categories with finite number of objects. Moreover, one of the algebras we study, called "backward finite category algebra", coincides with incidence algebras for combinatorically important cases originally studied in [9]. 
The purpose of this paper is to provide a new framework for the interplay between regions of mathematical sciences such as algebra, probability and physics, in terms of states as linear functional defined on category algebras. As is well known, quantum theory can be considered to be a noncommutative generalization of probability theory. At the beginning of quantum theory, matrix algebras played a crucial role (see [16] for example). In the present paper, we clarify that category algebras can be considered to be generalized matrix algebras and that the notions of states on categories as linear functionals defined on category algebras turns out to be a conceptual generalization of probability measures on sets as discrete categories (For the case of states on groupoid algebras over the complex field $\mathbb{C}$ it is already studied [17]).

Moreover, by establishing a generalization of famous GNS (Gelfand-Naimark-Segal) construction [18,19] (as for the studies in category theoretic context, see [20-22] for example), we obtain a representation of category algebras of ${ }^{\dagger}$-categories on certain generalized Hilbert spaces (semi-Hilbert modules over rigs), which can be considered to be an extension of the result in [17] for groupoid algebras over $\mathbb{C}$. This construction will provide a basis for the interplay between category theory, noncommutative probability and other related regions such as operator algebras or quantum physics.

Notation 1. In the present paper, categories are always supposed to be small (This assumption may be relaxed by applying some appropriate foundational framework). The set of all arrows in a category $\mathcal{C}$ is also denoted as $\mathcal{C} .|\mathcal{C}|$ denotes the set of all objects, which are identified with corresponding identity arrows, in $\mathcal{C}$. We also use the following notations:

$$
C^{\prime} \mathcal{C}_{C}:=\mathcal{C}\left(C, C^{\prime}\right), \mathcal{C}_{C}:=\sqcup_{C^{\prime} \in|\mathcal{C}|} \mathcal{C}\left(C, C^{\prime}\right),{ }^{\prime} \mathcal{C}:=\sqcup_{C \in|\mathcal{C}|} \mathcal{C}\left(C, C^{\prime}\right),
$$

where $\mathcal{C}\left(C, C^{\prime}\right)$ denotes the set of all arrows from $C$ to $C^{\prime}$.

\section{Category Algebras}

We introduce the notion of rig, module over rig, and algebra over rig in order to study category algebras in sufficient generality for various future applications in noncommutative probability, quantum physics and other regions of mathematical sciences such as tropical mathematics.

Definition 1 (Rig). A rig $R$ is a set with two binary operations called addition and multiplication such that

1. $R$ is a commutative monoid with respect to addition with the unit 0 ,

2. $R$ is a monoid with respect to multiplication with the unit 1 ,

3. $r^{\prime \prime}\left(r^{\prime}+r\right)=r^{\prime \prime} r^{\prime}+r^{\prime \prime} r,\left(r^{\prime \prime}+r^{\prime}\right) r=r^{\prime \prime} r+r^{\prime} r$ holds for any $r, r^{\prime}, r^{\prime \prime} \in R$ (Distributive law),

4. $0 r=0, r 0=0$ holds for any $r \in R$ (Absorption law).

Definition 2 (Module over Rig). A commutative monoid $M$ under addition with unit 0 together with a left action of $R$ on $M(r, m) \mapsto r m$ is called a left module over $R$ if the action satisfies the following:

1. $\quad r\left(m^{\prime}+m\right)=r m^{\prime}+r m,\left(r^{\prime}+r\right) m=r^{\prime} m+r m$ for any $m, m^{\prime} \in M$ and $r, r^{\prime} \in R$.

2. $0 m=0, r 0=0$ for any $m \in M$ and $r \in R$.

Dually we can define the notion of right module over $R$.

Let $M$ is left and right module over $R$. $M$ is called $R$-bimodule if

$$
r^{\prime}(m r)=\left(r^{\prime} m\right) r
$$

holds for any $r, r^{\prime} \in R$ and $m \in M$.

The left/right action above is called the scalar multiplication. 
Definition 3 (Algebra over Rig). A bimodule $A$ over $R$ is called an algebra over $R$ if it is also a rig with respect to its own multiplication which is compatible with scalar multiplication, i.e.,

$$
\left(r^{\prime} a^{\prime}\right)(a r)=r^{\prime}\left(a^{\prime} a\right) r,\left(a^{\prime} r\right) a=a^{\prime}(r a)
$$

for any $a, a^{\prime} \in A$ and $r, r^{\prime} \in R$.

Usually the term "algebra" is defined on rings and algebras are supposed to have negative elements. In this paper, we use the term algebra to mean the module over rig with multiplication.

Definition 4 (Category Algebra). Let $\mathcal{C}$ be a category and $R$ be a rig. An R-valued function $\alpha$ defined on $\mathcal{C}$ is said to be of backward (resp. forward) finite propagation if for any object $C$ there are at most finite number of arrows in the support of $\alpha$ whose codomain (resp. domain) is $C$. The module over $R$ consisting of all $R$-valued functions of backward (resp. forward) finite propagation together with the multiplication defined by

$$
\left(\alpha^{\prime} \alpha\right)\left(c^{\prime \prime}\right)=\sum_{\left\{\left(c^{\prime}, c\right) \mid c^{\prime \prime}=c^{\prime} \circ c\right\}} \alpha^{\prime}\left(c^{\prime}\right) \alpha(c), c, c^{\prime}, c^{\prime \prime} \in \mathcal{C}
$$

becomes an algebra over $R$ with unit $\epsilon$ defined by

$$
\epsilon(c)= \begin{cases}1 & (c \in|\mathcal{C}|) \\ 0 & (\text { otherwise })^{\prime}\end{cases}
$$

and is called the category algebra of backward (resp. forward) finite propagation $R_{0}[\mathrm{C}]\left(\right.$ resp. ${ }^{0} R[\mathcal{C}]$ ) of $\mathcal{C}$ over $R$. The algebra ${ }^{0} R_{0}[\mathcal{C}]$ over $R$ defined as the intersection $R_{0}[\mathcal{C}] \cap{ }^{0} R[\mathcal{C}]$ is called the category algebra of finite propagation of $\mathcal{C}$ over $R$.

Remark 1. ${ }^{0} R_{0}[\mathcal{C}]$ coincide with the algebra studied in [15] if $R$ is a ring.

In the present paper, we focus on the category algebras $R_{0}[\mathcal{C}],{ }^{0} R[\mathcal{C}]$ and ${ }^{0} R_{0}[\mathcal{C}]$ which are the same if $|\mathcal{C}|$ is finite, although other extensions or subalgebras of ${ }^{0} R_{0}[\mathcal{C}]$ are also of interest (see Examples 4 and 7).

Notation 2. In the following we use the term category algebra and the notation $R[\mathcal{C}]$ to denote either of category algebras $R_{0}[\mathrm{C}],{ }^{0} R[\mathcal{C}]$ and ${ }^{0} R_{0}[\mathcal{C}]$.

Definition 5 (Indeterminates). Let $R[\mathcal{C}]$ be a category algebra and $c \in \mathcal{C}$. The function $\chi^{\mathcal{c}} \in$ $R[\mathcal{C}]$ defined as

$$
\chi^{c}\left(c^{\prime}\right)= \begin{cases}1 & \left(c^{\prime}=c\right) \\ 0 & (\text { otherwise })\end{cases}
$$

is called the indeterminate (See Example 2) corresponding to $c$.

For indeterminates, it is easy to obtain the following:

Theorem 1 (Calculus of Indeterminates). Let $c, c^{\prime} \in \mathcal{C}, \chi^{c}, \chi^{c^{\prime}}$ be the corresponding indeterminates and $r \in R$. Then

$$
\begin{gathered}
\chi^{c^{\prime}} \chi^{c}= \begin{cases}\chi^{c^{\prime} \circ c} & \left(\operatorname{dom}\left(\mathrm{c}^{\prime}\right)=\operatorname{cod}(\mathrm{c})\right) \\
0 \quad & (\text { otherwise }),\end{cases} \\
r \chi^{c}=\chi^{c} r .
\end{gathered}
$$


In short, a category algebra $R[\mathcal{C}]$ is an algebra of functions on $\mathcal{C}$ equipped with the multiplication which reflects the compositionality structure of $\mathcal{C}$. By the identification of $c \in \mathcal{C} \mapsto \chi^{\mathcal{C}} \in R[\mathcal{C}]$, categories are included in category algebras.

Let us establish the basic notions for calculation in category algebras:

Definition 6 (Column, Row, Entry). Let $\alpha \in R[\mathcal{C}]$ and $C, C^{\prime} \in|\mathcal{C}|$. The elements $\alpha_{C}, C^{\prime}{ }, C^{\prime} \alpha_{C} \in$ $R[\mathcal{C}]$ defined as

$$
\begin{aligned}
\alpha_{C}(c) & = \begin{cases}\alpha(c) & \left(c \in \mathcal{C}_{C}\right) \\
0 & (\text { otherwise }),\end{cases} \\
C^{\prime} \alpha(c) & = \begin{cases}\alpha(c) & \left(c \in C^{\prime} \mathcal{C}\right) \\
0 & (\text { otherwise }),\end{cases} \\
C^{\prime} \alpha_{C}(c) & = \begin{cases}\alpha(c) & \left(c \in C^{\prime} \mathcal{C}_{C}\right) \\
0 & (\text { otherwise }),\end{cases}
\end{aligned}
$$

are called the $C$-column, $C^{\prime}$-row and $\left(C^{\prime}, C\right)$-entry of $\alpha$, respectively.

Please note that either of the data $\alpha_{C}(C \in|\mathcal{C}|), C^{\prime} \alpha\left(C^{\prime} \in|\mathcal{C}|\right)$ or ${ }^{C^{\prime}} \alpha_{C}\left(C, C^{\prime} \in|\mathcal{C}|\right)$ determine $\alpha$. Moreover, if $|\mathcal{C}|$ is finite,

$$
\alpha=\sum_{C, C^{\prime} \in|\mathcal{C}|} C^{\prime} \alpha_{C}
$$

By definition, the following theorem holds:

Theorem 2 (Polynomial Expression). For any $\alpha \in R[\mathcal{C}]$

$$
C^{\prime} \alpha_{C}=\sum_{c \in C^{\prime} \mathcal{C}_{C}} \alpha(c) \chi^{c}=\sum_{c \in C^{\prime} \mathcal{C}_{C}} \chi^{c} \alpha(c)
$$

If $|\mathcal{C}|$ is finite,

$$
\alpha=\sum_{c \in \mathcal{C}} \alpha(c) \chi^{c}=\sum_{c \in \mathcal{C}} \chi^{c} \alpha(c)
$$

The formulae above clarify that category algebras are generalized polynomial algebra (see Example 2). On the other hand, the following theorem, which shows that category algebras are generalized matrix algebras (see Example 7), also follows by definition:

Theorem 3 (Matrix Calculus). For any $\alpha, \alpha^{\prime} \in R[\mathcal{C}], C, C^{\prime} \in|\mathcal{C}|$ and $r \in R$, the followings hold:

$$
\begin{gathered}
\left(\alpha^{\prime}+\alpha\right)_{C}=\alpha_{C}^{\prime}+\alpha_{C}, C^{\prime}\left(\alpha^{\prime}+\alpha\right)={ }^{C^{\prime}} \alpha^{\prime}+{ }^{C^{\prime}} \alpha, \\
C^{\prime}\left(\alpha^{\prime}+\alpha\right)_{C}={ }^{C^{\prime}} \alpha_{C}^{\prime}+{ }^{C^{\prime}} \alpha_{C} \\
\left(r^{\prime} \alpha r\right)_{C}=r^{\prime} \alpha_{C} r, C^{\prime}\left(r^{\prime} \alpha r\right)=r^{\prime} C^{\prime} \alpha r, C^{\prime}\left(r^{\prime} \alpha r\right)_{C}=r^{\prime}{ }^{\prime} \alpha_{C} r \\
\left(\alpha^{\prime} \alpha\right)_{C}=\alpha^{\prime} \alpha_{C}=\sum_{C^{\prime \prime} \in|\mathcal{C}|} \alpha_{C^{\prime \prime}}^{\prime} C^{\prime \prime} \alpha_{C} \\
C^{\prime}\left(\alpha^{\prime} \alpha\right)={ }^{C^{\prime}} \alpha^{\prime} \alpha=\sum_{C^{\prime \prime} \in|\mathcal{C}|} C^{C^{\prime}} \alpha_{C^{\prime \prime}}^{\prime} C^{\prime \prime} \alpha \\
C^{\prime}\left(\alpha^{\prime} \alpha\right)_{C}=C^{C^{\prime}} \alpha^{\prime} \alpha_{C}=\sum_{C^{\prime \prime} \in|\mathcal{C}|} C^{\prime} \alpha_{C^{\prime \prime}}^{\prime} C^{\prime \prime} \alpha_{C} .
\end{gathered}
$$

The theorem above implies the following: 
Theorem 4. $\alpha \in R[\mathcal{C}]$ is determined by its action on columns $\epsilon_{C} /$ rows ${ }^{\prime} \epsilon$ of the unit $\epsilon$ for all $C, C^{\prime} \in|\mathcal{C}|$.

Proof. Let $\alpha \in R[\mathcal{C}]$ and $\epsilon$ be the unit of $R[\mathcal{C}]$. Then by definition

$$
\alpha=\alpha \epsilon, \alpha=\epsilon \alpha
$$

holds and it implies $\alpha_{C}=\alpha \epsilon_{C}, C^{\prime} \alpha=C^{\prime} \epsilon \alpha$, which determines $\alpha$.

Remark 2. It is convenient to make use of a kind of "Einstein convention" in physics: Double appearance of object indices which do not appear elsewhere means the sum over all objects in the category. For instance,

$$
C^{\prime}\left(\alpha^{\prime} \alpha\right)_{C}={ }^{\prime} \alpha_{C^{\prime \prime}}^{\prime} C^{\prime \prime} \alpha_{C}
$$

means

$$
C^{\prime}\left(\alpha^{\prime} \alpha\right)_{C}=\sum_{C^{\prime \prime} \in|\mathcal{C}|} C^{\prime} \alpha_{C^{\prime \prime}}^{\prime} C^{\prime \prime} \alpha_{C} .
$$

The notation is quite useful especially for category algebra $R[\mathcal{C}]$ where $|\mathcal{C}|$ is finite. In that case it is easy to show the decomposition of unit:

$$
\epsilon=\epsilon_{C}{ }^{C} \epsilon
$$

As a corollary,

$$
\alpha^{\prime} \alpha=\alpha^{\prime} \epsilon \alpha=\alpha^{\prime} \epsilon_{C}{ }^{C} \epsilon \alpha=\alpha_{C}^{\prime}{ }^{C} \alpha,
$$

holds, which means that the multiplication can be interpreted as inner product of columns and rows. Hence, you can insert ${ }_{C}{ }^{C}$ in formulae when $C$ does not appear elsewhere.

\section{Example of Category Algebras}

Let us see some important examples of category algebras.

Example 1 (Function Algebra). Let $\mathcal{C}$ be a set as discrete category, i.e., a category whose arrows are all identities. Then $R[\mathcal{C}]$ is nothing but the $R$-valued function algebra on $|\mathcal{C}|$, where the operations are defined pointwise.

When the rig $R$ is commutative such as $R=\mathbb{C}$, the function algebra is also commutative. On the other hand, a category algebra is in general noncommutative even if the rig is commutative. In this sense, category algebras can be considered to be generalized (noncommutative) function algebras.

As we have noted, category algebras can also be considered to be generalized polynomial algebras:

Example 2 (Monoid Algebra). Let $\mathcal{C}$ be a monoid, i.e., a category with only one object. Then $R[\mathcal{C}]$ is the monoid algebra of $\mathcal{C}$. For example, in the case of $\mathcal{C}=\mathbb{N}$ as additive monoid, $R[\mathcal{C}]$ is the polynomial algebra over $R$.

Since a monoid $\mathcal{C}$ has only one object, any $\alpha \in R[\mathcal{C}]$ can be presented as,

$$
\alpha=\sum_{c \in \mathcal{C}} \alpha(c) \chi^{c}
$$

by Theorem 2 which make it clear that $R[\mathcal{C}]$ is a generalized polynomial algebra.

As special cases of Example 2, we have group algebras.

Example 3 (Group Algebra). Let $\mathcal{C}$ be a group, i.e., a monoid whose arrows are all invertible. Then $R[\mathcal{C}]$ coincides with the group algebra of $\mathcal{C}$. For example, in the case of $\mathcal{C}=\mathbb{Z}, R[\mathcal{C}]$ is the Laurent polynomial algebra over $R$. 
By another generalization of Example 3 other than Example 2, we have groupoid algebras.

Example 4 (Groupoid Algebra). Let $\mathcal{C}$ be a groupoid, i.e., a category whose arrows are all invertible. When $|\mathcal{C}|$ is finite, $R[\mathcal{C}]$ is nothing but the groupoid algebra of $|\mathcal{C}|$. Otherwise $R[\mathcal{C}]$ is a unital extension of the groupoid algebra in conventional sense which is nonunital. $R[C]$ is quite useful to treat certain algebras which appeared in quantum physics [17]. (See Example 5 also.)

As special cases of the Example 4 we have matrix algebras:

Example 5 (Matrix Algebra). Let $\mathcal{C}$ be an indiscrete category, i.e., a category such that for every pair of objects $C, C^{\prime}$ there is exactly one arrow from $C$ to $C^{\prime}$. Denote the cardinal of $|\mathcal{C}|$ is $n$. Then $R[\mathcal{C}]$ is isomorphic to the matrix algebra $M_{n}(R)$.

Example 5 above shows that matrix algebras are category algebras. Conversely, any category algebra can be considered to be generalized matrix algebra (see Theorem 3 ). This point of view is also useful to study quivers [23], i.e., directed graphs with multiple edges and loops.

Example 6 (Path Algebra). Let $\mathcal{C}$ be the free category of a quiver $Q . R[\mathcal{C}]$ coincides with the notion of path algebra when the quiver $Q$ has finite number of vertices. Otherwise, the former includes the latter as a subalgebra.

Another important origin of the notion of category algebra is that of incidence algebra ([8,10-14], for example) originally studied on posets [9].

Example 7 (Incidence Algebra). Let $\mathcal{C}$ be a finely finite category [8], i.e., a category such that for any $c \in \mathcal{C}$ there exist finite number of pairs of arrows $c^{\prime}, c^{\prime \prime} \in \mathcal{C}$ satisfying $c=c^{\prime} \circ c^{\prime \prime}$. Then $R^{\mathcal{C}}$, the set of all functions from $\mathcal{C}$ to $R$, becomes a unital algebra and called the incidence algebra of $\mathcal{C}$ over R.

Let $\mathcal{C}$ be a category such that for any $C \in \mathcal{C}$ there exist at most finitely many arrows whose codomain is $C$. Then $R_{0}[\mathcal{C}]$ coincides with the incidence algebra on $\mathcal{C}$. (One of the most classical examples is the poset consisting of all positive integers ordered by divisibility). For the category satisfying the condition above, $R[\mathcal{C}]$ includes the zeta function $\zeta$ defined as

$$
\zeta(c)=1
$$

for all $c$. The multiplicative inverse of $\zeta$ is denoted as $\mu$ and called Möbius function. The relation $\mu \zeta=\zeta \mu=\epsilon$ is a generalization of the famous Möbius inversion formula, which has been considered to be the foundation of combinatorial theory since one of the most important papers in modern combinatorics [9].

\section{States on Categories}

We will introduce the notion of states on categories to provide a foundation for stochastic theories on categories. As we will see, we can construct noncommutative probability space, a generalized notion of measure theoretic probability space based on category algebras. The key insight is that what we need to establish statistical law is the expectation functional, which is the functional which maps each random variable (or "observable" in the quantum physical context) to its expectation value. Considering a functional on $R[C]$ as expectation functional, we can interpret $R[C]$ as an algebra of noncommutative random variables, such as observables of quanta.

Definition 7 (Linear Functional). Let $A$ be an algebra over a rig $R$. An R-valued linear function on $A$, i.e., a function preserving addition and scalar multiplication, is called a linear functional on 
A. A linear functional on $A$ is said to be unital if $\varphi(\epsilon)=1$ where $\epsilon$ and 1 denote the multiplicative unit in $A$ and $R$, respectively.

Definition 8 (Linear Functional on Category). Let $R$ be a rig and $\mathcal{C}$ be a category. A (unital) linear functional on $R[\mathcal{C}]$ is said to be an $R$-valued (unital) linear functional on the category $\mathcal{C}$.

Although the main theme here is stochastic theory making use of positivity structure defined later, linear functionals on category algebras are used not only in the context with positivity. A very interesting example is "umbral calculus" [24], an interesting tool in combinatorics, which can be interpreted as the theory of linear functionals on certain monoid algebras. Hence, studying the linear functionals on a category will lead to a generalization of umbral calculus.

Given a linear functional on a category, we obtain a function on the set of arrows. For categories with a finite number of objects, we can characterize the former in terms of the latter:

Theorem 5 (Linear Function and Function). Let $\varphi$ be a R-valued linear functional on $\mathcal{C}$. Then the function $\hat{\varphi}$ defined as

$$
\hat{\varphi}(c)=\varphi\left(\chi^{c}\right)
$$

becomes a $Z(R)$-valued function on $\mathcal{C}$, i.e., an R-valued function satisfying $r \hat{\varphi}(c)=\hat{\varphi}(c) r$ for any $c \in \mathcal{C}$ and $r \in R$. Conversely, when $|\mathcal{C}|$ is finite, any $Z(R)$-valued function $\phi$ on $\mathcal{C}$ gives $R$-valued linear functional $\check{\phi}$ defined as

$$
\check{\phi}(\alpha)=\sum_{c \in \mathcal{C}} \alpha(c) \phi(c)=\sum_{c \in \mathcal{C}} \phi(c) \alpha(c)
$$

and the correspondence is bijective.

Proof. Let $\varphi$ be a $R$-valued linear functional. Since $r \chi^{c}=\chi^{c} r$ for any $r \in R$ and $c \in \mathcal{C}$, we have $r \varphi\left(\chi^{c}\right)=\varphi\left(\chi^{c}\right) r$ which means $r \hat{\varphi}(c)=\hat{\varphi}(c) r$. The converse direction and bijectivity directly follows from definitions and Theorem 2 .

As a corollary we also have the following:

Theorem 6 (Unital Linear Functional and Normalized Function). Let $\mathcal{C}$ be a category such that $|\mathcal{C}|$ is finite. Then there is one to one correspondence between $R$-valued unital linear functionals $\varphi$ and normalized $Z(R)$-valued functions $\phi$ on $\mathcal{C}$, i.e., $Z(R)$-valued functions $\phi$ satisfying

$$
\sum_{C \in|\mathcal{C}|} \phi(C)=1 .
$$

(Please note that we identify objects and identity arrows.)

To define the notion of state as generalized probability measure which can be applied in noncommutative contexts such as stochastic theory on category algebras, we need the notions of involution and positivity structure.

Definition 9 (Involution on Category). Let $\mathcal{C}$ be a category. A covariant/contravariant endofunctor $(\cdot)^{\dagger}$ on $\mathcal{C}$ is said to be a covariant/contravariant involution on $C$ when $(\cdot)^{\dagger} \circ(\cdot)^{\dagger}$ is equal to the identity functor on $\mathcal{C}$. A category with contravariant involution which is identity on objects is called $a^{\dagger}$-category.

Remark 3. For the studies on involutive categories, which are categories with involution satisfying certain conditions, see [20,22] for example. 
Definition 10 (Involution on Rig). Let $R$ be a rig. An operation $(\cdot)^{*}$ on $R$ preserving addition and covariant/contravariant with respect to multiplication is said to be a covariant/contravariant involution on $R$ when $(\cdot)^{*} \circ(\cdot)^{*}$ is equal to the identity function on $R$. A rig with contravariant involution is called $a^{*}$-rig.

Definition 11 (Involution on Algebra). Let $A$ be an algebra over a rig $R$ with a covariant (resp. contravariant) involution $\overline{(\cdot)}$. A covariant (resp. contravariant) involution $(\cdot)^{*}$ on $A$ as a rig is said to be a covariant (resp. contravariant) involution on $A$ as an algebra over $R$ if it is compatible with scalar multiplication, i.e.,

$$
\left(r^{\prime} a r\right)^{*}=\overline{r^{\prime}} a^{*} \bar{r} \quad(\text { covariant case }), \quad\left(r^{\prime} a r\right)^{*}=\bar{r} a^{*} \overline{r^{\prime}} \quad(\text { contravariant case }) .
$$

An algebra A over $a^{*}$-rig $R$ with contravariant involution is called $a^{*}$-algebra over $R$.

Theorem 7 (Category Algebra as Algebra with Involution). Let $\mathcal{C}$ be a category with a covariant (resp. contravariant) involution $(\cdot)^{\dagger}$ and $R$ be a rig with a covariant (resp. contravariant) involution $\overline{(\cdot)}$. Then the category algebra ${ }^{0} R_{0}[\mathcal{C}]$ becomes an algebra with covariant involution (resp. *-algebra) over $R$.

Proof. The operation $(\cdot)^{*}$ defined as $\alpha^{*}(c)=\overline{\alpha\left(c^{\dagger}\right)}$ becomes a covariant (resp. contravariant) involution on ${ }^{0} R_{0}[\mathcal{C}]$. For the contravariant case,

$$
(\alpha \beta)^{*}(c)=\overline{\alpha \beta\left(c^{\dagger}\right)}=\overline{\sum_{c^{\dagger}=c^{\prime} \circ c^{\prime \prime}} \alpha\left(c^{\prime}\right) \beta\left(c^{\prime \prime}\right)}=\sum_{c^{\dagger}=c^{\prime} \circ c^{\prime \prime}} \overline{\alpha\left(c^{\prime}\right) \beta\left(c^{\prime \prime}\right)}=\sum_{c^{\dagger}=c^{\prime} \circ c^{\prime \prime}} \overline{\beta\left(c^{\prime \prime}\right)} \overline{\alpha\left(c^{\prime}\right)}
$$

which is equal to $\sum_{c=c^{\prime \prime}{ }^{\dagger} c^{\prime}} \overline{\beta\left(c^{\prime \prime}\right)} \overline{\alpha\left(c^{\prime}\right)}$. By changing the labels of arrows, it can be rewritten as

$$
\sum_{c=c^{\prime \prime}{ }^{\dagger} \circ c^{\prime}} \overline{\beta\left(c^{\prime \prime}\right)} \overline{\alpha\left(c^{\prime}\right)}=\sum_{c=c^{\prime} \circ c^{\prime \prime}} \overline{\beta\left(c^{\prime \dagger}\right)} \overline{\alpha\left(c^{\prime \prime}{ }^{\dagger}\right)}=\sum_{c=c^{\prime} \circ c^{\prime \prime}} \beta^{*}\left(c^{\prime}\right) \alpha^{*}\left(c^{\prime \prime}\right)=\beta^{*} \alpha^{*}(c) .
$$

The proof for the covariant case is similar and more straightforward.

Every category/rig has a trivial involution (identity). Thus, any category algebra ${ }^{0} R_{0}[\mathcal{C}]$ can be considered to be algebra with involution. In physics, especially quantum theory, the ${ }^{*}$-algebra ${ }^{0} R_{0}[\mathcal{C}]$ where $\mathcal{C}$ is a groupoid as ${ }^{+}$-category with inversion as involution and $R=\mathbb{C}$ as ${ }^{*}$-rig with complex conjugate as involution. (For the importance of groupoid algebra in physics, see [17] and references therein, for example).

Based on the involutive structure we can define the positivity structure on algebras:

Definition 12 (Positivity). A pair of rigs with involution $\left(R, R_{+}\right)$is called a positivity structure on $R$ if $R_{+}$is a subring such that $r, s \in R_{+}$and $r+s=0$ implies $r=s=0$, and that $a^{*} a \in R_{+}$ for any $a \in R$.

The most typical examples are $\left(\mathbb{C}, \mathbb{R}_{\geq 0}\right),\left(\mathbb{R}, \mathbb{R}_{\geq 0}\right)$, and $\left(\mathbb{R}_{\geq 0}, \mathbb{R}_{\geq 0}\right)$. Another interesting example is the tropical algebraic one $(\mathbb{R} \cup\{\infty\}, \mathbb{R} \cup\{\infty\})$ where $\mathbb{R} \cup\{\infty\}$ is considered to be a rig with respect to $\mathrm{min}$ and + .

Definition 13 (State). Let $R$ be a rig with involution and $\left(R, R_{+}\right)$be a positivity structure on $R$. A state $\varphi$ on an algebra $A$ with involution over $R$ with respect to $\left(R, R_{+}\right)$is a unital linear functional $\varphi: A \longrightarrow R$ which satisfies $\varphi\left(a^{*} a\right) \in R_{+}$and $\varphi\left(a^{*}\right)=\overline{\varphi(a)}$ for any $a \in R$, where $(\cdot)^{*}$ and $\overline{(\cdot)}$ denotes the involution on $A$ and $R$, respectively.

Remark 4. The last condition $\varphi\left(a^{*}\right)=\overline{\varphi(a)}$ follows from other conditions if $R=\mathbb{C}$. 
Definition 14 (Noncommutative Probability Space). A pair $(A, \varphi)$ consisting of an algebra $A$ with involution over a rig $R$ with involution and an $R$-valued state $\varphi$ is called a noncommutative probability space.

There are many studies on noncommutative probability spaces where the algebra $A$ is a ${ }^{*}$-algebra over $\mathbb{C}$. As is well known, the notion of noncommutative probability space essentially includes the one of probability spaces in conventional sense, which corresponds to the cases that algebras $A$ are commutative *-algebras (with certain topological structure). On the other hand, when the algebras are noncommutative, noncommutative probability spaces provide many examples which cannot be reduced to conventional probability spaces, such as models for quantum systems.

Definition 15 (State on Category). Let $R$ be a rig with involution and $\left(R, R_{+}\right)$be a positivity structure on $R$. A state on the category algebra ${ }^{0} R_{0}[\mathcal{C}]$ over $R$ with respect to $\left(R, R_{+}\right)$is said to be a state on a category $\mathcal{C}$ with respect to $\left(R, R_{+}\right)$.

As category algebras are in general noncommutative, states on categories provide many concrete noncommutative probability spaces generalizing such simplest examples as interacting Fock spaces [25] which are generalized harmonic oscillators, where the categories are indiscrete categories corresponding to certain graphs.

The notion of state can be characterized for the categories with finite number of objects as follows:

Theorem 8 (State and Normalized Positive Semidefinite Function). Let $\mathcal{C}$ be a category such that $|\mathcal{C}|$ is finite. Then there is one to one correspondence between states $\varphi$ with respect to $\left(R, R_{+}\right)$and normalized positive semidefinite $Z(R)$-valued functions $\phi$ with respect to $\left(R, R_{+}\right)$, i.e., normalized functions such that

$$
\sum_{\left\{\left(c, c^{\prime}\right) \mid \operatorname{dom}\left(\left(c^{\prime}\right)^{\dagger}\right)=\operatorname{cod}(c)\right\}} \overline{\xi\left(c^{\prime}\right)} \phi\left(\left(c^{\prime}\right)^{\dagger} \circ c\right) \xi(c)
$$

is in $R_{+}$for any function $\xi$ on $\mathcal{C}$ with finite support and that $\phi\left(c^{\dagger}\right)=\overline{\phi(c)}$, where $(\cdot)^{*}$ and $\overline{(\cdot)}$ denotes the involution on $A$ and $R$, respectively.

Proof. Please note that a function $\xi$ on $\mathcal{C}$ with finite support can be considered to be an element in ${ }^{0} R_{0}[\mathcal{C}]$ and vice versa when $|\mathcal{C}|$ is finite. Then the theorem follows from the identity

$$
\begin{aligned}
\xi^{*} \xi & =\left(\sum_{c^{\prime} \in \mathcal{C}} \overline{\xi\left(\left(c^{\prime}\right)^{\dagger}\right)} \chi^{c^{\prime}}\right)\left(\sum_{c \in \mathcal{C}} \chi^{c} \xi(c)\right) \\
& =\left(\sum_{c^{\prime} \in \mathcal{C}} \overline{\xi\left(c^{\prime}\right)} \chi^{\left(c^{\prime}\right)^{\dagger}}\right)\left(\sum_{c \in \mathcal{C}} \chi^{c} \xi(c)\right) \\
& =\sum_{\left\{\left(c, c^{\prime}\right) \mid \operatorname{dom}\left(\left(c^{\prime}\right)^{\dagger}\right)=\operatorname{cod}(c)\right\}} \overline{\xi\left(c^{\prime}\right)} \chi^{\left(c^{\prime}\right)^{\dagger} \circ c} \xi(c) .
\end{aligned}
$$

and the condition corresponding to $\varphi\left(\xi^{*}\right)=\overline{\varphi(\xi)}$.

The theorem above is a generalization of the result stated in Section 2.2.2 in [17] for groupoid algebras over $\mathbb{C}$. For the case of discrete category, the notion coincides with the notion of probability measure on objects (identity arrows). Hence, the notion of state on category can be considered to be noncommutative generalization of probability measure which is associated with the transition from set as discrete category (0-category) to general category (1-category). 
Given a state on $\mathrm{a}^{\dagger}$-category, we can construct a kind of GNS (Gelfand-Naimark-Segal) representation [18,19] (as for generalized constructions, see [20-22,26,27] for example) in a semi-Hilbert module defined below, a generalization of Hilbert space:

Definition 16 (Semi-Hilbert Module over Rig). Let $R$ be a rig with involution $\overline{(\cdot)}$. A right module E over $R$ equipped with a positive semidefinite sesquilinear form, i.e., a function $\langle\cdot \mid \cdot\rangle$ : $E \times E \longrightarrow R$ satisfying

$$
\begin{gathered}
\left\langle v^{\prime \prime} \mid v^{\prime} r^{\prime}+v r\right\rangle=\left\langle v^{\prime \prime} \mid v^{\prime}\right\rangle r^{\prime}+\left\langle v^{\prime \prime} \mid v\right\rangle r \\
\left\langle v^{\prime} \mid v\right\rangle=\overline{\left\langle v \mid v^{\prime}\right\rangle} \\
\langle v \mid v\rangle \in R_{+}
\end{gathered}
$$

for any $v, v^{\prime}, v^{\prime \prime} \in E$ and $r, r^{\prime} \in R$ is called a semi-Hilbert module over $R$.

When a semi-Hilbert module over $E$ is also a left module over $R$, the set $\operatorname{End}(E)$ consisting of module endomorphisms over $R$ on $E$ becomes an algebra over $R$ : The bimodule structure is given by $\left(r^{\prime} T r\right)(v)=r^{\prime} T(r v)$, where $T \in \operatorname{End}(E)$ and $r, r^{\prime} \in R$.

Theorem 9 (Generalized GNS Representation). Let $A$ be an ${ }^{*}$-algebra over a rig $R$ with involution $(\cdot)^{*}$. For any state $\varphi$ on $A$ with respect to $\left(R, R_{+}\right)$, there exist a semi-Hilbert module $E^{\varphi}$ over $R$ which is also a left $R$ module equipped with a positive semidefinite sesquilibear form $\langle\cdot \mid \cdot\rangle^{\varphi}$, an element $e^{\varphi} \in E^{\varphi}$ such that $\left\langle e^{\varphi} \mid e^{\varphi}\right\rangle^{\varphi}=1$, and a homomorphism $\pi^{\varphi}: A \longrightarrow \operatorname{End}\left(E^{\varphi}\right)$ between algebras over $R$ such that

$$
\varphi(\alpha)=\left\langle e^{\varphi} \mid \pi^{\varphi}(\alpha) e^{\varphi}\right\rangle^{\varphi}
$$

and

$$
\left\langle v^{\prime} \mid \pi^{\varphi}(\alpha) v\right\rangle^{\varphi}=\left\langle\pi^{\varphi}\left(\alpha^{*}\right) v^{\prime} \mid v\right\rangle^{\varphi}
$$

hold for any $\alpha \in A$ and $v, v^{\prime} \in E^{\varphi}$.

Proof. Let $E^{\varphi}$ be the algebra $A$ itself as a module over $R$ equipped with $\langle\cdot \mid \cdot\rangle^{\varphi}$ defined by $\left\langle\alpha^{\prime} \mid \alpha\right\rangle^{\varphi}=\varphi\left(\left(\alpha^{\prime}\right)^{*} \alpha\right)$. It is easy to show that $\langle\cdot \mid \cdot\rangle^{\varphi}$ is a positive semidefinite sesquilinear form and satisfies $\varphi(\alpha)=\left\langle e^{\varphi} \mid \pi^{\varphi}(\alpha) e^{\varphi}\right\rangle^{\varphi}$, and $\left\langle v^{\prime} \mid \pi^{\varphi}(\alpha) v\right\rangle^{\varphi}=\left\langle\pi^{\varphi}\left(\alpha^{*}\right) v^{\prime} \mid v\right\rangle^{\varphi}$ where $\pi^{\varphi}$ denotes the homomorphism $\pi^{\varphi}: A \longrightarrow \operatorname{End}\left(E^{\varphi}\right)$ defined by $\pi^{\varphi}(\alpha)=\alpha(\cdot)$, the left multiplication by $\alpha$, and $e^{\varphi}$ denotes the unit $\epsilon$ of $A$ as an element of $E^{\varphi}$.

Remark 5. When the rig $R$ is actually a ring, we can construct ${ }^{*}$-representation of $A$ as follows (This idea is due to Malte Gerhold): We call an endomorphism T on a semi-Hilbert module E adjointable if there is a (not necessarily unique) adjoint, i.e., an endomorphism $T^{*}$ with $\left\langle v^{\prime} \mid T v\right\rangle=$ $\left\langle T^{*} v^{\prime} \mid v\right\rangle$ for any $v, v^{\prime} \in E$. When $E$ is also a left $R$ module, the set of adjointable endomorphisms $\operatorname{Adj}(E)$ becomes a subalgebra over $R$ of End $(E)$. The set $\operatorname{Nul}(E)=\left\{T \mid\left\langle v^{\prime} \mid T v\right\rangle=0, \forall v, v^{\prime} \in E\right\}$ becomes a two-sided ideal in $\operatorname{Adj}(E)$. When $R$ is a ring, the quotient of $\operatorname{Adj}(E)$ by $\operatorname{Nul}(E)$ becomes $a^{*}$-algebra and we can construct the *-representation of $A$, since we can show that the two "adjoints" of an endomorphism coincide up to some element of $\mathrm{Nul}(E)$ by taking subtraction of endomophisms and can define the "taking adjoint" as involution operation in the quotient. In more general cases (especially for the rigs such that the cancellation law for addition does not hold), the GNS construction might not necessarily lead to ${ }^{*}{ }^{*}$-representations by adjointable endomorphisms.

When $A$ is a ${ }^{*}$-algebra over $\mathbb{C}$, we can prove Cauchy-Schwarz inequality for semiHilbert space. Then the set $N^{\varphi}=\left\{\alpha \in A \mid\langle\alpha \mid \alpha\rangle^{\varphi}=0\right\}$ becomes a subspace of $A$. By taking the quotient $E^{\varphi}=A / N^{\varphi}$, which becomes a pre-Hilbert space, we obtain the following "GNS (Gelfand-Naimark-Segal)" representation of $A$.

Theorem 10 (GNS Representation). Let $A$ be $a^{*}$-algebra over $\mathbb{C}$. For any state $\varphi$ on $A$ with respect to $\left(\mathbb{C}, \mathbb{R}_{\geq 0}\right)$, there exist a pre-Hilbert space $E^{\varphi}$ over $\mathbb{C}$ equipped with an inner product 
$\langle\cdot \mid \cdot\rangle^{\varphi}$, an element $e^{\varphi} \in E^{\varphi}$ such that $\left\langle e^{\varphi} \mid e^{\varphi}\right\rangle^{\varphi}=1$, and a homomorphism $\pi^{\varphi}: A \longrightarrow \operatorname{End}\left(E^{\varphi}\right)$ between algebras over $R$ such that

$$
\varphi(\alpha)=\left\langle e^{\varphi} \mid \pi^{\varphi}(\alpha) e^{\varphi}\right\rangle^{\varphi}
$$

and

$$
\left\langle v^{\prime} \mid \pi^{\varphi}(\alpha) v\right\rangle^{\varphi}=\left\langle\pi^{\varphi}\left(\alpha^{*}\right) v^{\prime} \mid v\right\rangle^{\varphi}
$$

hold for any $\alpha \in A$ and $v, v^{\prime} \in E^{\varphi}$.

By taking completion we have usual Hilbert space formulation popular in the context of quantum mechanics.

Remark 6. If the state $\varphi$ is fixed as "standard" one, such as "vacuum", the Dirac bracket notation becomes valid if we interpret as follows:

$$
|\alpha\rangle=\pi^{\varphi}(\alpha),\left\langle\alpha\left|=\varphi\left(\alpha^{*}(\cdot)\right),\left\langle\alpha^{\prime} \mid \alpha\right\rangle=\varphi\left(a^{*} b\right),\right| 0\right\rangle=|\epsilon\rangle \text { (vacuum). }
$$

As corollaries of theorems above, we have the following results, which are extensions of the Theorem 1 in [17]. :

Theorem 11 (Generalized GNS Representation of ${ }^{\dagger}$-Category). Let $\mathcal{C}$ be $a^{\dagger}$-category and $R$ be $a^{*}$-rig. For any $\varphi$ be a state on $\mathcal{C}$ with respect to $\left(R, R_{+}\right)$, there exist a semi-Hilbert module $E^{\varphi}$ over $R$ which is also a left $R$ module equipped with a sesquilinear form $\langle\cdot \mid \cdot\rangle^{\varphi}$, an element $e^{\varphi} \in E^{\varphi}$ such that $\left\langle e^{\varphi} \mid e^{\varphi}\right\rangle^{\varphi}=1$, and a homomorphism $\pi^{\varphi}:{ }^{0} R_{0}[\mathcal{C}] \longrightarrow \operatorname{End}\left(E^{\varphi}\right)$ between algebras over $R$ such that

$$
\varphi(\alpha)=\left\langle e^{\varphi} \mid \pi^{\varphi}(\alpha) e^{\varphi}\right\rangle^{\varphi}
$$

and

$$
\left\langle v^{\prime} \mid \pi^{\varphi}(\alpha) v\right\rangle^{\varphi}=\left\langle\pi^{\varphi}\left(\alpha^{*}\right) v^{\prime} \mid v\right\rangle^{\varphi}
$$

hold for any $\alpha \in A$ and $v, v^{\prime} \in E^{\varphi}$.

Theorem 12 (GNS Representation of ${ }^{\dagger}$-Category). Let $\mathcal{C}$ be $a^{\dagger}$-category. For any $\varphi$ be $a$ state on $\mathcal{C}$ with respect to $\left(\mathbb{C}, \mathbb{R}_{\geq 0}\right)$, there exist a pre-Hilbert space $E^{\varphi}$ over $\mathbb{C}$ equipped with an inner product $\langle\cdot \mid \cdot\rangle^{\varphi}$, an element $e^{\varphi} \in E^{\varphi}$ such that $\left\langle e^{\varphi} \mid e^{\varphi}\right\rangle^{\varphi}=1$, and a homomorphism $\pi^{\varphi}:{ }^{0} R_{0}[\mathcal{C}] \longrightarrow \operatorname{End}\left(E^{\varphi}\right)$ between algebras over $\mathbb{C}$ such that

$$
\varphi(\alpha)=\left\langle e^{\varphi} \mid \pi^{\varphi}(\alpha) e^{\varphi}\right\rangle^{\varphi}
$$

and

$$
\left\langle v^{\prime} \mid \pi^{\varphi}(\alpha) v\right\rangle^{\varphi}=\left\langle\pi^{\varphi}\left(\alpha^{*}\right) v^{\prime} \mid v\right\rangle^{\varphi}
$$

hold for any $\alpha \in A$ and $v, v^{\prime} \in E^{\varphi}$.

Funding: This work was partially supported by Research Origin for Dressed Photon, JSPS KAKENHI (grant number 19K03608 and 20H00001) and JST CREST (JPMJCR17N2).

Institutional Review Board Statement: Not applicable.

Informed Consent Statement: Not applicable.

Acknowledgments: The author is grateful to Hiroshi Ando, Soichiro Fujii and Misa Saigo for fruitful discussions and comments.

Conflicts of Interest: The author declares no conflict of interest.

\section{References}

1. Eilenberg, S.; MacLane, S. General Theory of Natural Equivalences. Trans. Am. Math. Soc. 1945, 58, 231-294. [CrossRef]

2. Mac Lane, S. Categories for the Working Mathematician, 2nd ed.; Springer: New York, NY, USA, 1998. 
3. Awodey, S. Category Theory, 2nd ed.; Oxford University Press: New York, NY, USA, 2010.

4. Leinster, T. Basic Category Theory; Cambridge University Press: Cambridge, UK, 2014.

5. Accardi, L. Quantum Probability: An Historical Survey. Contemp. Math. 2000, 261, 145-159.

6. Hora, A.; Obata, N. Quantum Probability and Spectral Analysis of Graphs; Springer: Berlin/Heidelberg, Germany, 2007.

7. Biane, P.; Guionnet, A.; Voiculescu, D.-V. Noncommutative Probability and Random Matices at Saint-Flour; Springer: Berlin/Heidelberg, Germany, 2012.

8. Leinster, T. Notions of Möbius inversion. Bull. Belg. Math. Soc. Simon Stevin 2012, 19, 909-933. [CrossRef]

9. Rota, G.-C. On the foundations of combinatorial theory I: theory of Möbius functions. Z. Wahrscheinlichkeitstheorie Verw. Gebiete 1964, 2, 340-368. [CrossRef]

10. Cartier, P.; Foata, D. Problémes Combinatoires de Commutation et Réarrangements; No. 85 in Springer Lecture Notes in Mathematics; Springer: Berlin New York, NY, USA, 1969.

11. Leroux, P. Les catégories des Möbius. Cah. Topol. Géom. Différ. Catégor. 1975, 16, 280-282.

12. Content, M.; Lemay, F.; Leroux, P. Catégories de Möbius et fonctorialités: un cadre général pour l'inversion de Möbius. J. Combin. Theory Ser. 1980, 10, 142-149. [CrossRef]

13. Haigh, J. On the Möbius algebra and the Grothendieck ring of a finite category. J. Lond. Math. Soc. 1980, 21, 81-92. [CrossRef]

14. Lawvere, F.W.; Mehni, M. The Hopf algebra of Möbius intervals. Theory Appl. Categ. 2010, 24, 221-265.

15. Mitchel, B. Rings with several objects. Adv. Math. 1972, 8, 1-161. [CrossRef]

16. Born, M. Natural Philosophy of Cause and Chance; Clarendon: Oxford, UK, 1949.

17. Ciaglia, F.M.; Ibort, A.; Marmo, G. Schwinger's Picture of Quantum Mechanics III: The statistical Interpretation. Int. J. Geom. Meth. Modern Phys. 2019, 16, 1950165. [CrossRef]

18. Gelfand, I.; Neumark, M. On the imbedding of normed rings into the ring of operators in Hilbert space. Mat. Sb. 1943, 12, 187-217.

19. Segal, I. Irreducible representations of operator algebras. Bull. Am. Math. Soc. 1947, 53, 73-88. [CrossRef]

20. Jacobs, B. Involutive Categories and Monoids, with a GNS-Correspondence. Found. Phys. 2012, 42, 874-895. [CrossRef]

21. Parzygnat, A.J. From Observables and States to Hilbert Space and Back: A 2-Categorical Adjunction. Appl. Categ. Struct. 2018, 26, 1123-1157. [CrossRef]

22. Yau, D. Involutive Category Theory; No. 2279 in Springer Lecture Notes in Mathematics; Springer: Cham, Switzerland, 2020.

23. Gabriel, P. Unzerlegbare Darstellungen. I. Manuscripta Math. 1972, 6, 71-103. [CrossRef]

24. Roman, S.; Rota, G.-C. The umbral calculus. Adv. Math. 1978, 27, 95-188. [CrossRef]

25. Accardi, L.; Bożejko, M. Interacting Fock spaces and gaussianization of probability measures. Infin. Dimens. Anal. Quantum Probab. Relat. Top. 1998, 1, 663-670. [CrossRef]

26. Steinspring, W.F. Positive functions on $C^{*}$-algebras. Proc. Amer. Math. Sci. 1955, 6, 211-216.

27. Paschke, W.L. Inner product modules over B*-algebras. Trans. Amer. Math. Soc. 1973, 182, 443-468. [CrossRef] 